\title{
IDENTIFICACIÓN POR MÉTODOS MOLECULARES DE ADENOVIRUS ASOCIADOS A CONJUNTIVITIS
}

\author{
IDENTIFICATION OF ADENOVIRUS ASSOCIATED WITH \\ CONJUNCTIVITIS BY MOLECULAR METHODOLOGY
}

\author{
MEJÍA-LÓPEZ $\mathrm{H}^{1}$, MATÍAS-FLORENTINO M², VÉLEZ-MONTOYA R ${ }^{3}$
}

\begin{abstract}
RESUMEN
Objetivo: Por medio de métodos moleculares como la Reacción en Cadena de la Polimerasa (PCR) y la secuenciación del material genético, identificar adenovirus en pacientes con conjuntivitis.

Métodos: Se procesaron muestras de raspado del saco conjuntival inferior de 51 pacientes (39 diagnosticados con conjuntivitis folicular y 12 diagnosticados con conjuntivitis vernal) para identificar adenovirus por medio de la PCR genérica. Todas las muestras positivas en la PCR genérica, fueron cultivadas en células VERO para el aislamiento del virus, éste se demostró por inmunofluorescencia. Se utilizó la PCR múltiple para la caracterización de los serotipos aislados y las variantes genéticas se identificaron mediante la secuenciación automatizada.

Resultados: Veintiocho de los pacientes diagnosticados con conjuntivitis folicular y seis de los diagnosticados con conjuntivitis vernal resultaron positivos a adenovirus (67\%). El cultivo en células VERO permitió el aislamiento del virus de 8 muestras. Sólo tres de los aislados fueron identificados (un serotipo Ad1 y dos Ad2) por la PCR múltiple que identifica adenovirus del subgénero $C$. Por
\end{abstract}

\begin{abstract}
Objective: To identify adenovirus in patients with conjunctivitis by molecular methods such as the Polymerase Chain Reaction (PCR) and DNA sequencing.

Methods: Samples of scrapings from the inferior fornix of 51 patients (39 diagnosed with Follicular Conjunctivitis and 12 diagnosed with Vernal Conjunctivitis) were processed by generic PCR to identify adenovirus. All the samples that were PCR positive were cultured on VERO cells for virus isolation, with this being demonstrated by immunofluorescence. For the identification of the isolated serotype, the multiplex PCR was utilized and DNA automated sequencing was employed to identify the genetic variants.

Results: Twenty-eight of the individuals diagnosed with Follicular Conjunctivitis and six of those diagnosed with Vernal Conjunctivitis, had positive results to adenovirus (67\%). The cultures in VERO cells allowed the isolation of eight samples. Only three of the isolated viruses (one Ad1 and two Ad2) were identified by the multiplex PCR used to identify the subgenus $\mathrm{C}$ adenovirus. An Ad1 genetic variant was identified by automated sequencing
\end{abstract}

Recibido: 20/10/05. Aceptado: 19/7/06.

Instituto de Oftalmología Conde de Valenciana. Unidad de Investigación. México D.F. México.

1 Licenciada en Ciencias Biomédicas.

2 Técnico de Laboratorio.

3 Doctor en Medicina.

Correspondencia:

Chimalpopoca 14 Col. Obrera

Del. Cuauhtemoc. CP 06800

México, D.F.

México

E-mail: linchm10@yahoo.com 
secuenciación automatizada se identificó una variante genética correspondiente al Ad1, mientras que los aislados de Ad2 fueron idénticos a los reportados en el Banco de Genes.

Conclusiones: La Reacción en Cadena de la Polimerasa y la Secuenciación son herramientas útiles para la identificación y caracterización de agentes causantes de enfermedades tales como la Conjuntivitis por Adenovirus .

Palabras Clave: Conjuntivitis folicular, conjuntivitis vernal, adenovirus, epidemiología molecular, infecciones oculares. while the Ad2 serotypes were identical to the ones reported by Genbank.

Conclusions: The Polymerase Chain Reaction and DNA sequencing are useful tools to identify and characterize microorganisms responsible for diseases such as conjunctivitis caused by adenoviruses (Arch Soc Esp Oftalmol 2006; 81: 375-382).

Key words: Follicular conjunctivitis, vernal conjunctivitis, adenovirus, molecular epidemiology, ocular infections.

\section{INTRODUCCIÓN}

Los adenovirus (Ad) están ampliamente distribuidos en el mundo, infectan millones de personas causando diversas manifestaciones clínicas tales como queratoconjuntivitis, cistitis hemorrágica, gastroenteritis y afectan seriamente vías respiratorias altas y bajas. Las enfermedades por adenovirus varían de esporádicas a epidémicas sin producir signos o síntomas patognomónicos, son usualmente agudas y autolimitadas, pero en niños muy pequeños pueden ser fatales o estar asociados con daños crónicos. Se conocen más de 100 serotipos y se han identificado 6 subgéneros (A-F), basados en varias características biológicas y moleculares, sin embargo, se han reportado 51 de ellos capaces de infectar al hombre $(1,2)$. Las infecciones que producen estos virus son íntimamente relacionadas con su tropismo en diverso tejidos; Ad8, Ad19 y Ad37 infectan ojo Ad1, Ad2, Ad3, Ad4, Ad5 y Ad6 infectan preferentemente vías respiratorias mientras que $\operatorname{Ad} 40$ y Ad41 causan infección en tracto digestivo (3).

Numerosos reportes muestran un amplio rango de andenovirus pertenecientes a los subgeneros B (Ad3 y Ad7), C (Ad1, Ad2, y Ad5), o E (Ad4), como causantes esporádicos de conjuntivitis, conjuntivitis folicular o fiebre faringo-conjuntival (46). Brotes epidémicos son producidos por el subgenero D fundamentalmente por Ad8 causando queratoconjuntivitis (7). Por estas razones y debido a que en México no se conocen reportes que aporten datos sobre su dinámica epidemiológica, resulta importante la rápida identificación del virus fundamentalmente en casos en donde las formas características no están presentes. Diversa metodología se emplea en la actualidad para la identificación de adenovirus. El aislamiento en líneas celulares apropiadas es de gran utilidad en la formación de bancos genómicos en caso de brotes epidémicos, sin embargo, requiere de 2 a 4 semanas para su identificación (8). La Reacción en Cadena de la Polimerasa (PCR), es una técnica moderna, extraordinariamente específica, sensible y rápida, que requiere de una muestra pequeña del especimen en estudio (9), por estas razones en el presente trabajo utilizamos la PCR para analizar muestras de pacientes que acudieren a la consulta externa de nuestro instituto y que fueron diagnosticados con conjuntivitis vernal o conjuntivitis folicular. Una modificación, la PCR múltiple fue empleada para la identificación de serotipos responsables de la infección; este rubro es de nuestro particular interés debido a que ciertos serotipos afectan preferentemente al ojo y al respecto no existen reportes en México. Con el fin de conocer las variantes genotípicas, estudiamos la secuenciación de tres serotipos aislados.

\section{SUJETOS MATERIAL Y MÉTODOS}

\section{Muestras clínicas}

Fueron analizadas muestras de pacientes con conjuntivitis vernal o conjuntivitis folicular, de octubre de 2004 a agosto de 2005. La toma de la muestra se realizó haciendo un raspado de saco conjuntival inferior utilizando un hisopo de arginato de calcio, el cual se introdujo posteriormente, en un medio de trasporte conteniendo $50 \mathrm{mg} / \mathrm{ml} \mathrm{de}$ gentamicina, $500 \mathrm{U}$ de penicilina-estreptomici- 
$\mathrm{na} / \mathrm{ml}$, fungizona $1 \mathrm{mg} / \mathrm{ml}$ y $5 \%$ albúmina sérica bobina en un buffer de Hank.

\section{Extracción de DNA}

La extracción de ADN se realizó con el mini kit QIAamp según protocolo de Quiagen (QIAGEN, Sciences. Maryland, USA). El material genético fue almacenado a $-20^{\circ} \mathrm{C}$ hasta su identificación.

\section{Aislamiento de adenovirus}

Las muestras biológicas fueron inoculadas en células epiteliales de riñón de mono verde (VERO), una línea celular susceptible a ser infectada por Ad. Después de 3 a 5 pases en las células VERO (células epiteliales de riñón de mono verde), hasta la obtención del efecto citopático característico, se realizó la prueba de inmunofluorescencia directa (IF) (10), utilizando el Kit de Chemicon (Chemicon International Inc.). Como colorante de contraste fue empleado yoduro de propídio el cual tiñe de color rojo al material genómico. Todos los cultivos positivos en la prueba de inmunofluorescencia, fueron procesados para la PCR genérica para adenovirus, con el fin de confirmar el aislamiento.

\section{PCR genérica}

Los oligonucleótidos ADRJC1 y ADRJC2 fueron usados para amplificar un producto de PCR de 140pb (tabla I). Este amplificado corresponde a una región altamente conservada del ADN que codifica para la proteína que constituye a los capsómeros de todos los adenivirus. Como controles se usaron Ad1, Ad2 y Ad5, aislados de pacientes con Infec- ción Respiratoria Aguda, previamente caracterizados. Cada reacción fue hecha según el protocolo de Elnifro EM et al. (11). La reacción se realizó en un termociclador GeneAmp PCR System 2400 (Perkin Elmer Co. Norwalk, Connecticut). Los productos de PCR se analizaron en geles de agarosa al 1,5\% teñidos con bromuro de etidio $(1 \mu \mathrm{g} / \mathrm{ml})$. Se usó como marcador estándar de pesos moleculares el Ready-Load 100 bp DNA Ladder (Invitrogen, L.T.).

\section{PCR múltiple}

Serotipos específicos fueron identificados por un PCR múltiple, se usó para ello, olinonucleótidos que reconocen el exón que codifica para el filamento de los adenovirus Ad1, Ad2 y Ad5 del subgénero C. El oligonucleótido corriente arriba AdCF, es común en los tres Ad y los oligonucleótidos corriente abajo Ad1R, Ad2R y Ad5R pertenecen al ADN que codifica el filamento los serotipo específicos, estos permiten la obtención de los amplicones de 630pb, 204pb y 455pb, respectivamente (tabla I). Fueron usados como controles Ad1, Ad2 y Ad5, aislados de pacientes con Infección Respiratoria Aguda, previamente caracterizados. Cada reacción fue hecha según protocolo Adhikary AK et al. (12). La reacción se llevo a cabo en un termociclador GeneAmp PCR System 2400 (Perkin Elmer Co. Norwalk, Connecticut, USA). Los productos de amplificación fueron separados en un gel de agarosa al $1,5 \%$, posteriormente se extrajeron del gel según protocolo de QUIAEX II. (QIAGEN Sciences. Maryland, USA); la pureza y concentración de los mismos, fueron verificadas en un gel de agarosa al $1,5 \%$, teñido con bromuro de etidio $(1 \mu \mathrm{g} / \mathrm{ml})$. Como marcador estándar de pesos moleculares se usó el Low Mass Ladder (Invitrogen, L.T.).

Tabla I. Oligonucleótidos genéricos y serotipo-específicos

\begin{tabular}{llll}
\hline & Nombre & Secuencia (5'-3') & Tamaño del amplicón (pb) \\
\hline Genérica & ADRJC1 & 5'-GACATGACTTTCGAGGTCGATCCCATGGA-3' & 140 \\
& ADRJC2 & 5'-CCGGCTGAGAAGGGTGTGCGCAGGTA-3' & \\
Serotipo & & 5'-TGCTTGCGCTHAAAATGGGCA-3' & 630 \\
Ad1 & AdCF & 5'-CGAGTATAAGACGCCTATTTACA-3' & 204 \\
Ad2 & Ad2R & 5'-CGCTAAGAGCGCCGCTAGTA-3' & 455 \\
Ad5 & Ad5R & 5'-ATGCAAAGGAGCCCCGTAC-3' & \\
\hline \hline
\end{tabular}

ADRJC1 y ADRJC2 corresponden a una secuencia del gen que codifica para la proteína exón de los capsómeros de Ad (24). AdCF es común en el subgenero C y Ad1R, Ad2R y Ad5R son olinonucleótidos serotipo específicos. 


\section{Secuenciación de serotipos}

Para la secuenciación de los adenovirus que fueron identificados en el PCR múltiple, se realizó una PCR con los oligonucleótidos que identifican los serotipos del subgénero $\mathrm{C}$ (CF, 1R y 2R) y se utilizó el kit BigDye Terminator (Applied Biosystems) que contiene cada ddNTP marcado con fluorescencia, el volumen de reacción fue de $10 \mu \mathrm{L}$ que contenía 10ng del ADN a secuenciar y $1 \mu \mathrm{M}$ de cada primer. Las condiciones de amplificación fueron a $96^{\circ} \mathrm{C} / 30$ seg seguido de 25 ciclos a $50^{\circ} \mathrm{C} / 15 \mathrm{seg}$ y $60^{\circ} \mathrm{C} / 4 \mathrm{~min}$. Los productos de la reacción fueron purificados en Columnas CentriSep (Princeton Separations) y analizados en un secuenciador ABI Prism 310. Los electroferogramas obtenidos fueron comparados con secuencias de nucleótidos reportadas en el Banco de Genes, AB108423 para Ad1 y AJ278920 para Ad2.

\section{RESULTADOS}

\section{PCR genérica}

Fueron analizadas 51 muestras de raspado de saco conjuntival inferior de pacientes de consulta externa. La tabla II muestra la lista de todos los pacientes estudiados y el resultado de la PCR en cada paciente. Resultaron positivos 28 , que representan el $75 \%$ de los individuos diagnosticados con conjuntivitis folicular y 6 , que representan el 50\% de los diagnosticados con conjuntivitis vernal, en la figura 1 se muestran el amplificado de $140 \mathrm{pb}$, representativo de un resultado positivo, obtenido con los oligonucleótidos genéricos.

\section{Aislamiento de adenovirus}

Todas las muestras positivas a la PCR genérica, fueron sembradas en cultivos de células VERO. Fueron aisladas 8 de las muestras inoculadas. La figura 2a muestra células VERO sin infectar, en la figura $2 \mathrm{~b}$ se observa el efecto citopático característico de las células infectadas con el virus. Como pruebas confirmatorias del aislamiento, se utilizó la técnica de IF directa. En la figura 3 se presenta un ejemplo de la IF directa en células infectadas, en las que pueden apreciarse las inclusiones virales de color amarillo brillante.

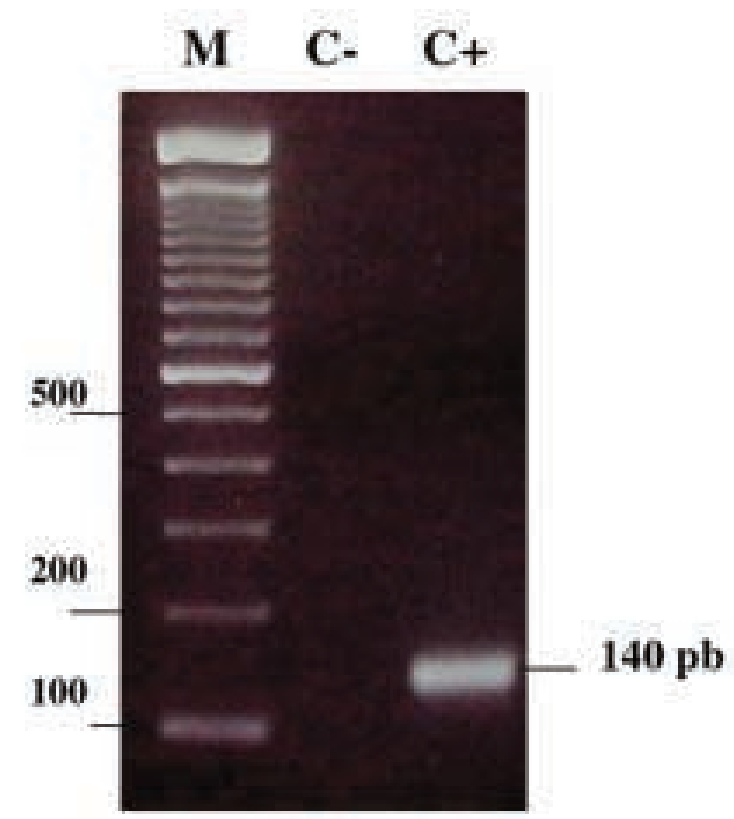

Fig. 1: Gel 1,5\% de agarosa teñidos con bromuro de etidio que representa un ejemplo de amplificación del PCR genérico con ADRJC1 y ADRJC2. M, marcador estándar Ready-Load 100 bp DNA; 2, control negativo; 3, amplificado de $140 \mathrm{pb}$.

\section{PCR múltiple}

Los aislados fueron probados con oligonucleótidos que identifican serotipos específicos del subgenero $\mathrm{C}$ de adenovirus. Tres muestras de las ocho aisladas, pudieron ser reconocidas en esta prueba. La figura 4 muestra los productos de amplificación obtenidos y puede observarse un amplificado de $630 \mathrm{pb}$ y dos de 204pb correspondientes a Ad1 y Ad2 respectivamente, al ser comparados con el control positivo de la reacción, el cual muestra los pro-

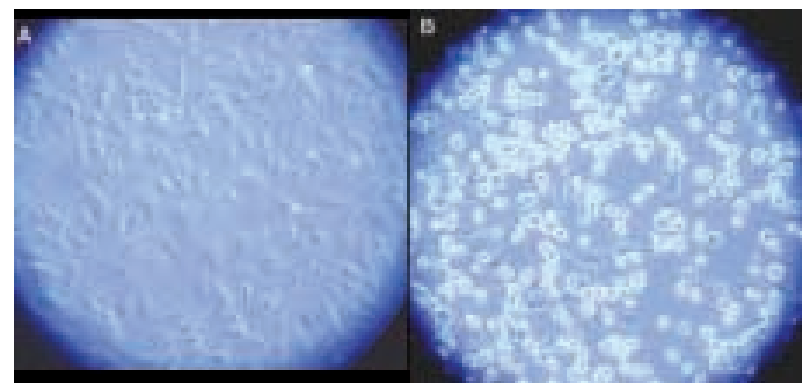

Fig. 2: Cultivos de Células Vero. A) células no infectadas. B) Efecto citopático característico de adenovirus. 
Tabla II. Individuos estudiados por PCR genérica y PCR múltiple serotipo-específico para adenovirus

\begin{tabular}{|c|c|c|c|c|c|c|c|c|c|}
\hline Paciente & Dx & $\begin{array}{l}\text { PCR Ad } \\
\text { genérico }\end{array}$ & $\begin{array}{c}\text { Aislado en } \\
\text { Células Vero. }\end{array}$ & $\begin{array}{l}\text { PCR Serotipo } \\
\text { específico }\end{array}$ & Paciente & Dx & $\begin{array}{l}\text { PCR Ad } \\
\text { genérico }\end{array}$ & $\begin{array}{c}\text { Aislado en } \\
\text { Células Vero. }\end{array}$ & $\begin{array}{c}\text { PCR Serotipo } \\
\text { específico }\end{array}$ \\
\hline JRC & $\mathrm{CV}$ & + & - & ND & ALN & $\mathrm{CF}$ & - & - & ND \\
\hline GOC & $\mathrm{CV}$ & - & - & ND & MOT & $\mathrm{CF}$ & - & - & ND \\
\hline CARB & $\mathrm{CV}$ & + & - & ND & RM & $\mathrm{CF}$ & - & - & ND \\
\hline NPV & $\mathrm{CV}$ & - & - & ND & PVJ & $\mathrm{CF}$ & - & - & ND \\
\hline JAC & $\mathrm{CV}$ & + & - & ND & BSF & $\mathrm{CF}$ & - & - & ND \\
\hline LRVT & $\mathrm{CV}$ & - & - & ND & EAA & CF & + & + & ND \\
\hline IMVS & $\mathrm{CV}$ & + & - & ND & AAM & $\mathrm{CF}$ & + & + & Ad2 \\
\hline JARM & $\mathrm{CV}$ & - & - & ND & MBP & $\mathrm{CF}$ & - & - & ND \\
\hline EMP & $\mathrm{CV}$ & + & - & ND & MBS & $\mathrm{CF}$ & - & - & ND \\
\hline ENC & $\mathrm{CV}$ & - & - & ND & APR & $\mathrm{CF}$ & - & - & ND \\
\hline CRMR & $\mathrm{CV}$ & + & - & ND & MSM & $\mathrm{CF}$ & - & - & ND \\
\hline OGE & $\mathrm{CV}$ & - & - & ND & MGS & CF & + & + & ND \\
\hline ANP & CFA & + & - & ND & HIERV & $\mathrm{CF}$ & + & - & ND \\
\hline KSJR & $\mathrm{CM}$ & + & - & ND & LDP & $\mathrm{CF}$ & + & - & ND \\
\hline XSM & CF & + & - & ND & BVR & CF & + & - & ND \\
\hline DMC & $\mathrm{CF}$ & + & - & ND & SCA & $\mathrm{CF}$ & + & - & ND \\
\hline JJMV & Blefaritis & + & - & ND & SHL & $\mathrm{CF}$ & + & - & ND \\
\hline EYU & $\mathrm{CF}$ & + & + & ND & SJTL & $\mathrm{CF}$ & + & - & ND \\
\hline CMC & $\mathrm{CF}$ & - & - & ND & DJA & $\mathrm{CF}$ & + & - & ND \\
\hline MGT & $\mathrm{CF}$ & + & - & ND & RMIG & $\mathrm{CF}$ & + & - & ND \\
\hline GAF & $\mathrm{CF}$ & + & + & Ad1 & GCV & $\mathrm{CF}$ & + & - & ND \\
\hline EGSG & $\mathrm{CF}$ & + & + & ND & OHL & $\mathrm{CF}$ & + & - & ND \\
\hline JGNM & $\mathrm{CF}$ & + & - & ND & AMG & $\mathrm{CF}$ & + & - & ND \\
\hline SGZ & $\mathrm{CF}$ & + & + & ND & JTT & $\mathrm{CF}$ & + & - & ND \\
\hline DMS & $\mathrm{CF}$ & - & - & ND & MSMC & CF & + & - & ND \\
\hline FBR & $\mathrm{CF}$ & + & + & $\mathrm{Ad} 2$ & & & & & \\
\hline
\end{tabular}

Dx= Diagnóstico; $\mathrm{CV}=$ Conjuntivitis vernal; $\mathrm{CF}=$ Conjuntivitis folicular; $\mathrm{CFA}=$ Conjuntivitis folicular aguda y $\mathrm{CM}=$ Conjunctivitis membranosa.

ductos amplificados pertenecientes a Ad1, Ad2 y Ad5, este último de $455 \mathrm{pb}$.

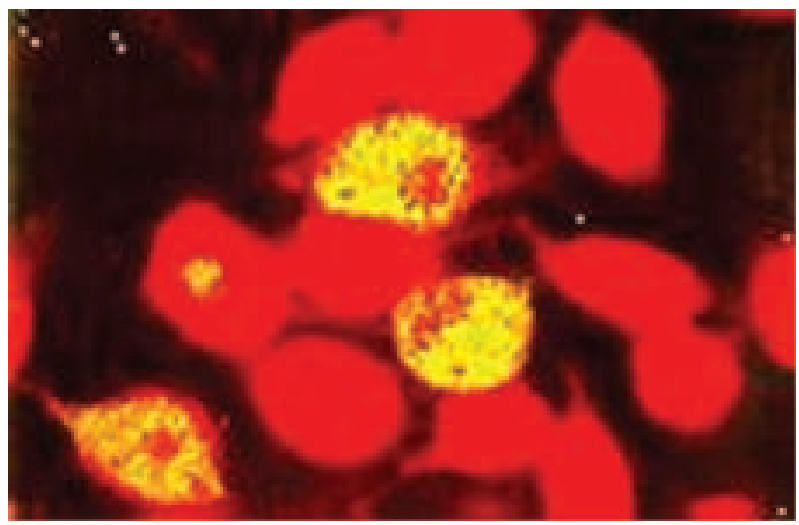

Fig. 3: Inmunofluorescencia directa en células Vero infectadas con una muestra de raspado conjuntival y teñidas con anticuerpos específicos contra adenovirus (kit de Chemicon Co.). inclusiones virales de color amarillo brillante. Las células no infectadas aparecen de color rojo.

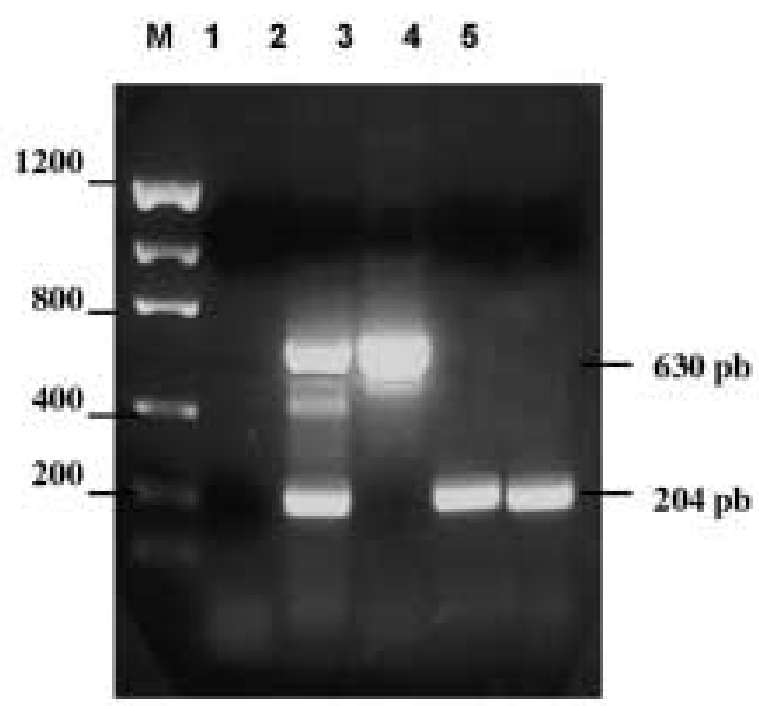

Fig. 4: Gel 1,5\% de agarosa teñidos con bromuro de etidio. M, marcador estándar Low Mass Ladder (Invitrogen, L.T.); Amplificación del PCR múltiple del subgénero $C$ en: 2, control positivo; 3 , control negativo; 4, 5 y 6 los aislados de los pacientes GAF, FBR y AAM, respectivamente. 


\section{ACTTTACAACAACAAT}

\section{ACTTTACAACAGCAAT}

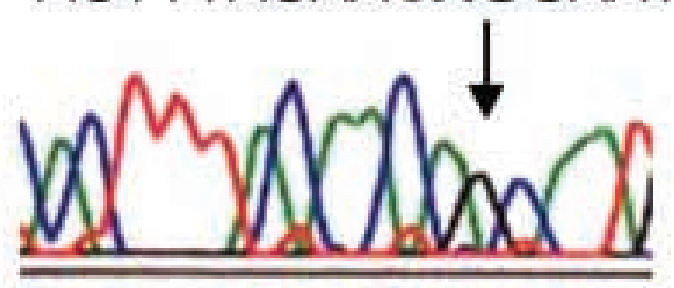

Fig. 5: Análisis de la secuencia del amplificado obtenido con AdCF/AdIR (Ad1). Se observa un cambio de A por $G$ en el nucleótido 199 (flecha) el cual implica un cambio en el codón CAA que codifica para serina (panel I, secuencia encontrada en AB108423 del Banco de Genes), a uno CAG que codifica para asparagina (panel II).

\section{Secuenciación de serotipos}

Los tres serotipos identificados en la PCR múltiple fueron secuenciados. La figura 5 muestra la porción del electroferograma perteneciente a Ad1 en donde se encontró un cambió de A por $\mathrm{G}$ en el nucleótido 199 (flecha) el cual implica un cambio en el codón CAA que codifica para serina (en la secuencia encontrada en AB108423), a un CAG que codifica para asparagina. Las secuencias de los serotipos Ad2, fueron idénticas a las reportadas en la dirección AJ278920, par los amplificados obtenidos con los nucleótidos AdCF/Ad2R.

\section{DISCUSIÓN}

En México Pizarro et al., identificaron adenovirus respiratorios usando las técnicas de fijación de complemento y neutralización (13-15). Por otra parte, no existen reportes sobre la prevalencia y dinámica epidemiológica de adenovirus que afectan al ojo. Por esta razón en el presente estudio se reporta el uso de técnicas modernas altamente específicas, sensibles y rápidas, que requieren de muy poco material biológico para identificar adenovirus como uno de los agentes más frecuentes en las infecciones oculares.

En nuestro reporte se pueden distinguir dos grupos, uno diagnosticado como conjuntivitis vernal y otro como conjuntivitis folicular. El 50\% del primer grupo se encontró infectado con adenovirus y un paciente de este grupo (dato no mostrado) presentó herpes simple tipo 1 (HSV-1). La queratoconjuntivitis vernal es una de las formas de la alergia crónica en ojos que se presenta en niños, en donde hay una disminución visual debido al daños en la córnea. Recientemente Zhan et al. mostraron el papel inmunomodulador de las células de epitelio conjuntival en la expresión de moléculas coestimuladoras (16). También se ha demostrado una participación importante de la respuesta de tipo TH2 (17). Una incógnita a resolver en la conjuntivitis vernal, plantea el hecho de que si la infección induce la permanencia del cuadro clínico en esta alergia, puesto que los virus comprometen a una respuesta de tipo celular. Muy pocos estudios se han hecho al respecto, sin embargo, es crucial mencionar que en cuadros respiratorios los adenovirus tienen una participación en la transducción de los RNAm de la célula infectada, evitando la síntesis de enzimas inducida por interferón, permitiendo así, la replicación del virus al inicio de la infección. Por otro lado, Un producto del gen E3 de trascripción temprana del virus, induce la internalización y degradación del receptor para el factor de crecimiento epidermal (EGF-R). El EGF-R, es una proteína transmembranal con actividad de tirosina cinasa, que al ser estimulado por su ligando, induce la fase de síntesis celular. Otra proteína codificada por E3 antagoniza la respuesta inflamatoria mediada por TNF- $\alpha$. Estos mecanismos facilitan la persistencia del virus en las células infectadas $(18,19)$.

Las manifestaciones clínicas en el ojo por infección con adenovirus pueden incluir conjuntivitis folicular aguda, bulbar o palpebral, con una quemosis que causa dolor y alteraciones de la visión, también puede haber formación de membranas. Esta enfermedad está frecuentemente acompañada de linfoadenopatía auricular significativa; pueden ser partes de un síndrome respiratorio faríngeo (fiebre faringoconjuntival) o presentarse como una entidad separada. El $76 \%$ de los pacientes captados en este estudio fueron diagnosticados con conjuntivitis folicular, sin embargo, estos síntomas no son patognomónicos de una infección por adenovirus, otros patógenos pudieron ser la causa del cuadro como son, citomegalovirus, enterovirus, o coxsakie virus (20). 
Debido a que los adenovirus tienen un tropismo marcado por células epiteliales de los tejidos que infectan, es de crucial importancia la identificación de los serotipos que circulan en nuestra población; con este fin, utilizamos una prueba PCR-múltiple con la que hemos podido definir la presencia de Ad1 y Ad2, puesto que se han mostrado con alta frecuencia serotipos del subgénero $\mathrm{C}$ produciendo daños oftalmológicos $(4,5)$. En este reporte, se muestra el aislamiento del virus a partir de cultivo en células VERO y la caracterización de 2 serotipos pertenecieron al subgénero $\mathrm{C}$. Es fundamental el estudio de un mayor número de casos, para comprender la dinámica epidemiológica de este virus en México.

La secuenciación automatizada es una herramienta moderna y muy útil en el estudio del material genético, aquí permitió mostrar que la PCR múltiple fue sensible en la identificación de los serotipos Ad1 y Ad2 como causantes de la infección y además, nos aporta datos para reconocer variantes genéticas que pudieran tener relevancia en el ámbito clínico.

La secuenciación de los Ad1 y Ad2 identificados por PCR múltiple, corresponde al amplificado del exón que codifica el filamento de estos serotipos y que los identifica específicamente (12). Resulta interesante, que el subgénero $\mathrm{C}$ de los adenovirus tiene un genoma íntimamente relacionado y se han reportado en el banco de genes varias secuencias de su genoma, lo que de alguna manera habla de su enorme diversidad. La variante encontrada en el Ad1 aislado, refleja lo reportado en la literatura sobre el polimorfismo de este grupo tan especial (21). Un mayor número de muestras permitirá conocer los serotipos circulantes en México y por medio de su secuenciación poder identificar las variantes antigénicas.

De este trabajo se puede concluir que la reacción en cadena de la polimerasa y la secuenciación son herramientas útiles para la identificación y caracterización de agentes causantes de enfermedades tales como la conjuntivitis por adenovirus y son muchos más sensibles que la técnica de aislamiento por cultivo en células VERO.

\section{BIBLIOGRAFÍA}

1. Schmitz H, Wigand R, Heinrich W. Worldwide epidemiology of human adenovirus infections. Am J Epidemiol 1983; 117: 455-466.
2. Horwitz MS. Adenoviruses. In: Fields BN, Kinipe DM, Howley PM, Chanock RM, Melnick JL, Monath TP, et al. Fields Virology. III Ed. Philadelphia: Lippincott Raven Publishers; 1996; 2149-2166.

3. De Jong JC, Wermenbol AG, Verweij-Uijterwaal MW, Slaterus KW, Wertheim-Van Dillen P, et al. Adenoviruses from human immunodeficiency virus-infected individuals, including two strains that represent new candidate serotypes Ad50 and Ad51 of species $B 1$ and D, respectively. $J$ Clin Microbiol 1999; 37:3940-3945.

4. Cooper RJ, Yeo AC, Bailey AS, Tullo AB. Adenovirus polymerase chain reaction assay for rapid diagnosis of conjunctivitis. Invest Ophthalmol Vis Sci 1999; 40: 90-95.

5. Takeuchi S, Itoh N, Uchio E, Aoki K, Ohno S. Serotyping of adenoviruses on conjunctival scrapings by PCR and sequence analysis. J Clin Microbiol 1999; 37: 1839-1845.

6. Shepetiuk SK, Norton R, Kok T, Irving LG. Outbreak of adenovirus type 4 conjunctivitis in South Australia. J Med Virol 1993; 41: 316-318.

7. Jernigan JA, Lowry BS, Hayden FG, Kyger SA, Conway $B P$, Gröschel DH, et al. Adenovirus type 8 epidemic keratoconjunctivitis in an eye clinic: risk factor and control. $J$ Infect Dis 1993; 167: 1307-1313.

8. Green M. Oncogenic viruses. Annu Rev Biochem 1970; 39: 701-756.

9. Elnifro EM, Ashshi AM, Cooper RJ, Klapper PE. Multiplex PCR: optimization application in diagnostic virology. Clin Microbiol Rev 2000; 13: 559-570.

10. Wood SR, Sharp IR, Caul EO, Paul I, Bailey AS, Hawkins $M$, et al. Rapid detection and serotyping of adenovirus by direct immunofluorescence. J Med Virol 1997; 51: 198201.

11. Elnifro EM, Cooper RJ, Klapper PE, Bailey AS. PCR and restriction endonuclease analysis for rapid identification of human adenovirus subgenera. J Clin Microbiol 2000; 38: 2055-2061.

12. Adhikary AK, Inada T, Banik U, Numaga J, Okabe N. identification of subgenus $C$ adenoviruses by fiber-based multiplex PCR. J Clin Microbiol 2004; 42: 670-673.

13. Pizarro Suarez E, Ortega PO. Investigación de anticuerpos hacia los adenovirus. II Anticuerpos específicos de tipo. Rev Latinoamer Microbiol 1962; 5: 153-159.

14. Pizarro E, Reséndiz Lara J, Quintero Mármol E, Arreguín Macín L. Investigación de anticuerpos hacia los adenovirus. III. Estudio en dos comunidades cerradas. Rev Latamer Microbiol Parasitol 1966; 8: 9-12.

15. Pizarro E, Reséndiz Lara J. Aislamiento de adenovirus tipo 2. Estudio de un brote en casa de cuna. Rev Invest Salud Publica (Méx) 1967; 27: 233-244.

16. Zhan H, Towler HM, Calder VL. The immunomodulatory role of human conjunctival epithelial cells. Invest Ophthalmol Vis Sci 2003; 44: 3906-3910.

17. Leonardi A, DeFranchis G, Zancanaro F, Crivellari $G, D e$ Paoli M, Plebani M, et al. Identification of local Th2 and ThO lymphocytes in vernal conjunctivitis by cytokine flow cytometry. Invest Ophthalmol Vis Sci 1999; 40: 30363040.

18. Kitajewski J, Schneider RJ, Safer B, Munemitsu SM, Samuel CE, Thimmappaya B, et al. Adenovirus VAI RNA antagonizes the antiviral action of interferon by preventing activation of the interferon-induced eIF-2alpha kinase. Cell 1986; 45: 195-200. 
19. Carlin CR, Tollefson AE, Brady HA, Hoffman FL, Wold WS. Epidermal growth factor receptor is down-regulated by a 10,400 MW protein encoded by the E3 region of adenovirus. Cell 1989; 57: 135-144.

20. Shulman LM, Manor Y, Azar R, Handsher R, Vonsover A, Mendelson E, et al. Identification on a new strain of fasti- dious enterovirus 70 as the causative agent of an outbreak of hemorrhagic conjunctivitis. J Clin Microbiol 1997; 35: 2145-2149.

21. Adrian T. Genome polymorphysm of human adenoviruses of subgenus C. Arch Virol 1996; 141:10211031. 